at last sank into a torpid state; it was often moaning; the pupils were dilated, the heart laboring, there was frequent micturition, the respiration exceedingly laborious, and, in short, there was every mark of plethora, or over-fulness of the vascular? system. In the course of two hours from this period the animal began to recover; and in about three hours these symptoms had subsided; considerable languor remained. The animal took a large meal twice or thrice in twenty-four hours, and after each meal precisely similar effects were presented. The animal became more feeble daily. In a month after the operation it died.

"Experiment II. I next removed the spleen from another dog, but instead of giving full meals, as in the last experiment, I gave a small quantity of food every one or two hours. The animal ate voraciously; no unpleasant symptoms resulted. This plan was pursued for three weeks, when the animal to all appearance was quite well; in fact, it became fat; the ligature on the splenic artery had come away, and the wound in the abdomen was healed. I then commenced giving full meals twice in twenty-four hours; the same train of symptoms followed each meal, and at the same period, as in the last experiment, though perbaps not so urgent. The animal died in a month from the commencement of this plan of feeding."

Is the spleen to be considered a useless organ, because an animal of the canine species can live after it has been removed? Are the resources of nature in obviating the effects of diseased organs to be denied to have an existence, because they do not come under the cognizance of our senses, or, in the present state of our knowledge, do not admit of an explanation? Because life can be maintained when the liver is wanting, is it a proof that this organ is of no use in the animal economy? Is the bile not necessary to digestion because its secretion is suspended in abscesses of the liver? There is a period in our existence when not a single organ in the three cavities, nor a bloodvessel, nor a lymphatic, nor a nerve, is developed. Hence it may be concluded, by a parity of reason, that every organ, except those that are vital, may cease to perform its function for a considerable length of time without destroying life.

\title{
REMARKS ON DISEASES OF THE PELVIC VISCERA.
}

[Communicated for the Boston Medical and Surgical Journal.]

I HAVE seen several cases of abscesses which formed in the appendages of the uterus, particularly in the ovaria, which were opened, and the patients recovered in all the cases I can recollect. Likewise I have been concerned in several cases of an enlargement of the uterus, which were considered scirrhous or cancerous, and of course were pronounced incurable by the attending physicians. In some of these cases the uterus was as large as in the sixth or seventh month of gestation; exceeding hard, attended with heat, pain, hectic fever, and great emaciation; yet these patients have often recovered. Scirrhous affections of the uterus*

* The fact would seem to be that there is an indurated state of the uterus which is not very liable to terminate in cancer; though there are other forms of this disease which onen do.
the the 
do not seem so dangerous as other scirrhosities. Several cases of dropsy of the ovaria, in which $\mathbf{l}$ have been concerned, have proved fatal. In this affection would it be advisable to tap and inject wine, as in hydrocele ? Though I should think it more advisable to introduce a tube after tapping, and thereby excite inflammation and adhesion, as Larrey recommends in hydrocele. I think this method much more safe than to inject wine; and as likely to effect a permanent cure. Why is it that we find it so difficult to cure encysted dropsy, by internal treatment? Is it because it is in a great measure out of the course of the general circulation? In the early stage of hydrocele, I have given equal parts of digitalis, emetic tartar, and calomel, which has in several instances removed the disease. Might not a similar treatment sometimes prove successful in the early stage of ovarian dropsy? I might here state several cases of polypus of the uterus; but Dr. T. Chadbourne has treated this subject so ably that it is wholly unnecessary.

In fistula in ano $\mathbf{l}$ have nothing interesting to remark, save in one case where the ulcer penetrated the coats of the rectum and vagina, and had several pipes, one of which terminated in the perineum, and the others near the orifice of the vagina. The disease was of long standing; the patient was discouraged and melancholy. In the treatment of this disease I was much perplexed; to divide the coats of the rectum and vagina, and make them one cavity, was, for obvious reasons, not warrantable; and merely to palliate and let my patient sink, my conscience would not admit. I thought of making use of the method recommended by the French surgeons, viz., to introduce a lead wire ; and by gradually twisting it, it would cut its way through the parts, and these being in near contact would adhere about as fast as they were divided by the wire ; but my patient was of a scrofulous habit, and the ulcer was in a bad condition, which deterred me from trying the experiment at this time. After once operating by incision, however, the cure was so tedious that I prepared for the above operation; but my patient refused, so instead of laying the vagina and rectum in one, I carried the incision from the ulcer in the vagina and rectum obliquely between their coats, laterally and backwards two thirds of an inch to the right of the rectum, dividing the sphincter ani its whole width.* I apprehended great trouble, as I expected she would not be able to retain the feces; but it was not the case, though she said she could not command the involuntary discharge of air and liquid, \&c., for some days. The cure in this case was extremely tedious; I salivated, made use of stimulating injections, gave balsam copaiva, quinine, iodine, \&c. But her recovery was eventually perfect. She has now enjoyed good health for several years. - In the year 1825 I was called to a patient of Dr. Samuel Smith's, of Bristol, who by a fall on the stake of a hay-cart, received a wound which lacerated the rectum and bladder. The wound in the bladder was at least two inches in length; so that hay and feces passed from the rectum into the bladder. The mode of treatment in this case was to introduce a catheter of elastic gum into the bladder, and let it there

- Here the young surgeon will not misunderstand me; no portion of the vagina should by any moan bo divided. Of this we must be certain. 
remain till the wound in it should be healed; as the wound of the bladder did not correspond with that of the rectum when the bladder was empty, and by drawing off the water once every half hour by means of a small syringe introduced into the catheter, the bladder was kept in a collapsed state till the wound was healed, which required about one month. But after the wound was healed, another difficulty occurred, which threatened to render abortive all that had been done; there were seeds of grass left in the bladder, which had formed nuclei for small calculi, from the size of a pigeon shot to that of a duck shot. The procedure which Dr. Smith adopted in this case was to seek for a solvent for these stones; and he found that the marine acid, when largely diluted, was the most perfect solvent; it dissolved all the stony part, and left them merely a soft pulp. By injecting the marine acid, properly diluted, into the bladder, and retaining it as long as could conveniently be borne, and then removing it by a small syringe, the catheter still remaining in the bladder, this difficulty was removed, and the patient finally restored to a comfortable state of health. Query: Why could not a treatment similar to this be adopted in the early stages of gravel ?

I have had occasion to operate in several cases of imperforate rectum, all of which proved fatal, save one; though in every instance the child seemed much relieved by the operation.

Stricture of the Rectum.- In the year 1822 I was called, in consultation, to a case considered to be colic. The colon was swollen as large as a man's arm. The integuments of the abdomen were so extended as plainly to show the track and shape of the colon. Several strong cathartics were given, but to no effect; the patient died about the third day. Leave being obtained, the body was examined; but what was supposed to have been colic, was found to be a stricture of the rectum. 'The woman, some years prior to this illness, while walking across a rough, rocky field, fell on a stone. The blow was on the superior part of the sacrum. After this she was troubled with pain and inflammation of the part, which finally terminated in an abscess, and was discharged by the rectum. She afterwards occasionally had pain in the back, and discharges of pus per anum. But I do not know that she consulted any physician, or made use of any remedy. Nothing of this was known to any of her attending physicians till after her death. On the examination of the parts by dissection, the superior part of the rectum, for about two inches, was found to be hard and thickened, the calibre of which was so diminished that it would scarcely admit the point of the little finger; on the superior part of this stricture, there was a fungous excrescence, which shut down and closed the stricture like a valve. Had this woman's case been known, her life doubtless might have been prolonged, and possibly the disease removed.

In another case of stricture of the rectum, in which I was called in consultation, the patient's health was much impaired; she had hectic fever, night sweats, \&c. There was ulceration of the rectum, which was fistulous, the fistula terminating in the perineum. In this case the stricture was about three inches up the rectum; the walls of the rectum 
were much thickened, rather hard and callous; and above this thickening there was a strong, tendinous band, which formed a circle, almost obliterating the calibre of the rectum. The mode of operating in this case was by introducing the fore finger of the right hand into the rectum, and penetrating the stricture (which would hardly admit of a pipe-stem) with the point of the finger, which was effected with considerable difficulty; then with the left hand a probe was introduced by the fistulous opening in the perineum, till felt in the rectum by the fore finger of the right hand; then by an assistant, a small, well-shaped director was introduced into the rectum, guided by the probe, which was still kept there, till it was felt by the fore finger of the right hand. The probe was then withdrawn; and the operator, taking the director with the thumb and fore finger of his left hand, and gently pressing its point against the point of the index finger of the right hand, and the part of the director in the left being firmly supported by bringing down its point by the fore finger of the right hand, all that portion of the rectum below the ulcer, with the stricture, was made to protrude and appear externally. The parts were now examined as they laid on the director, and the fistula and stricture freely divided: but as the coats of the rectum were much thickened, hard and callous, it was necessary to bring down other portions of the tendinous ring and divide them. A bougie of three fourths of an inch was first used, and afterwards of an inch diameter.

This mode of operating was first suggested to me by the late Dr. Nathan Smith, formerly professor of Anatomy and Surgery at Dartmouth College. But it should never be done except by very gentle means; it is much safer to push the rectum upwards than to pull it downwards. We cannot well operate in this manner more than three inches up the rectum ; in the superior part it would be extremely difficult and hazardous.

Franklin, N. H., March 23d, 1840.

JoB WILSON.

BOSTON MEDICAL AND SURGICAL JOURNAL.

BOSTON, A PRIL $29,1840$.

\section{CLASS BOOK OF ANATOMY.}

Mr. R. S. DAvs has published, the past week, a fourth revised edition of the Class Book of Anatomy. Although originally designed for schools, where its utility has been long established, students of medicine, and even those who have no particular desire to retain anything more than an elementary knowledge of the science on which is based the practice of physic and surgery, have become its distinguished patrons. The special object of the author, when the work first appeared in 1834, is thus expressed in the preface: "Should it, in the hands of public instructers, be instrumental in explaining to the young a general knowledge of their own curious organization, it may lead to the adoption of such habits in early life as will insure health in youth and intellectual vigor in age."

An impression that anatomy should be studied, like other useful sub- 